\title{
The Two Couriers Problem
}

\author{
William Gilreath \\ william.gilreath@protonmail.com
}

Submitted: 6 June 2018

Revised: 15 May 2019

\begin{abstract}
The Two Couriers Problem is an algebra problem, originally stated in 1746 by the French mathematician Clairaut. For over a century, the Two Couriers Problem has been re-used in various forms as a mathematical problem, in textbooks and journals, by different mathematicians and authors.

The Two Couriers Problem involves cases where division by zero arises in practice, where each has a real-world, actual result for the solution. Thus the Two Couriers Problem is a centuries old algebra problem with actual applied results that involve division by zero. It is an excellent mathematical problem to evaluate different methods for dividing by zero.

Division by zero has many different mathematical approaches. Conventional mathematics handles division by zero as an indeterminate or undefined result. Transmathematics defines division by zero as either nullity or explicitly positive or negative infinity. Two other approaches are by Saitoh, who defines division by zero simply as zero, and Barukčić who defines division by zero as either unity or explicitly positive or implicitly negativity infinity. The question is, which approach is best to solve the mathematical problem of division by zero?

The paramount goal of this paper is to use the Two Couriers Problem as an objective test to examine and evaluate mathematical approaches to division by zero - and find which one is best.
\end{abstract}

\section{Introduction}

The Two Couriers Problem is a long standing problem in algebra that concerns the position of two couriers who are sent on a journey, one after the other. The problem involves division by zero and yet has a very clear interpretation in the real world - we are never in any doubt about the actual position of the couriers. This problem, therefore, provides an excellent test bed for examining different approaches to division by zero. 


\section{The Two Couriers Problem}

\subsection{History of the Two Couriers Problem}

The earliest known statement of the Two Couriers Problem is by the French mathematician Alexis-Claude Clairaut in his didactic work Élémens D' Algbre [6] published in 1746. We gain some insight into the origins of the Two Couriers Problem when Clairaut states, in the preface to Élémens D' Algbre, that he wants to complete his didactic works with an application of algebra to geometry. Since that time, the The Two Couriers Problem has been used repeatedly, in different textbooks, from Clairaut, 1746, to the 1913 textbook Advanced Algebra by Joseph Victor Collins [7], giving a timeline of almost two centuries.

\subsection{The Problem Statement}

Over the centuries, the Two Couriers Problem has been stated in different books, with different definitions, but essentially the same concept.

Clairaut originally put the problem of the two couriers as, "Un Courrier est parti d'un lieu, il y a 9 heures et fait 5 lieues en 2 heures, on enovoye un autre Courrier après lui, dont la vitesse est telle qu'il fait 11 lieues en 3 heurers; Il s'agit de sçavoir ou ce second un Courrier attrapera le premier."

This may be translated as, "A courier departed from a place 9 hours ago and traveled 5 leagues in 2 hours, another courier was sent after him, the speed of which was 11 leagues in 3 hours; it's about knowing where this second courier will catch the first." The anonymous translators for the present paper note that the French word ou, translated as where, is ambiguous. It can mean a place in space or in time or both. They warn that his ambiguity might be important in the analysis of the Two Couriers Problem.

De Morgan [1] defines the problem of the two couriers as, "Two couriers, A and B, in the course of a journey between towns C and D, are the same moment of time at $\mathrm{A}$ and $\mathrm{B}$. A goes m miles, and B, $\mathrm{n}$ miles an hour. At what point between $\mathrm{C}$ and $\mathrm{D}$ are they together? Let the distance $\mathrm{AB}$ be called a."

The definition given by de Morgan is a more general, but unambiguously spatial, statement of the problem. We use de Morgan's arithmetical solutions in our analysis and evaluation of the Two Couriers Problem.

\subsection{The Six Cases}

Speaking of the two couriers, De Morgan [1] states, "It is evident that the answer depends upon whether they are going in the same or opposite directions, where A goes faster or slower than B, and so on. But all these, as we shall see, are include in the same general problem ..."

Note point $\mathrm{A}$ and $\mathrm{B}$ along the line between $\mathrm{C}$ and $\mathrm{D}$ are the points where the couriers are located at the same moment of time.

There are six cases in the Two Couriers Problem, which are: 
Case first: Suppose they are going in the same direction from C to D, and that $\mathrm{m}$ is greater than $\mathrm{n}$.

Case second: Suppose them now moving in the same direction as before, but let B move faster than A.

Case third: If they are moving from $\mathrm{D}$ to $\mathrm{C}$, and if $\mathrm{B}$ moves faster than A.

Case fourth: Similarly if they are moving from D to C, and A moves faster than B. Similar 1st case.

Case fifth: Suppose them now moving in contrary directions...they must now meet somewhere between $\mathrm{A}$ and $\mathrm{B}$.

Case sixth: Let them be moving in contrary directions, but let $\mathrm{A}$ be moving towards $\mathrm{C}$, and B towards D. They will then have met somewhere between $\mathrm{A}$ and $\mathrm{B}$.

The general expression for the first four cases of when in time the two couriers will meet is where $a$ is the distance between the two couriers: $a /(m-n)$ or $a /(n-m)$.

For the last two cases the expression of the time when the two couriers will meet is: $a /(m+n)$.

\subsection{Analysis}

The significance of the Two Couriers Problem is that division by zero occurs in circumstances where we are in no doubt about the position of the two couriers. Thus, we have a real-word explanation of what division by zero means in these easy to visualise cases.

There are two cases of division by zero in the first four cases of the problem. Case 1: when $a>0$ and $m=n$ the result, involving division by zero, is $a / 0$. In this case the couriers are apart and will never meet because they are moving at the same speed, which may be zero. Case 2: when $a=0$ and $m=n$ the result, involving division by zero, is $0 / 0$. In this case the two couriers start in the same place and remain together in two different circumstances. Case 2.1: when $m=n=0$ the two couriers are not moving and remain in their one, original, place. Case 2.2: when $m=n \neq 0$ the couriers start off together and remain together as they sweep out a half-infinite line or, at least, a segment of a half-infinite line that lies on CD.

Similarly there are two cases of division by zero in the fifth and sixth cases. Case 3 when $a>0$ and $m=n=0$ the result is $a / 0$. In this case the couriers are apart and will never meet because they are moving at zero speed. This case is entirely subsumed in Case 1 . Finally there is Case 4: when $a=0$ and $m=n=0$ the result is $0 / 0$. In this case the two couriers start in the same place and remain in their one, original, place. This case is entirely subsumed in Case 2.1.

Thus we have three cases. In Case 1 we have $a / 0$, implying that the couriers are apart and never meet. Cases 2.1 and 2.2 both have $0 / 0$ and are the same temporally, the two couriers are together at all points in time, but these cases differ spatially. In Case 2.1 the two couriers are at a single point for all time but in Case 2.2 the couriers sweep out a halfinfinite line over time. Thus our analysis differs depending on whether we take a spatial, temporal or spatiotemporal interpretation of the problem. 
However, in all cases, $a / 0$ means the couriers are apart for all time and $0 / 0$ means the couriers are together for all time.

\subsection{Standard Mathematics}

In standard mathematics $0 / 0$ and $a / 0$ are not numbers; they are not objects in mathematics. But in metamathematics $0 / 0$ is said to be $i n$ determinate [5], and $a / 0$ is said to be undefined [2]. This meta theory gives us just enough information to distinguish Case 1 and Case 2, but not enough information to distinguish Case 2.1 and 2.2.

\subsection{Transmathematics}

Transmathematics [3] defines the numbers nullity and both positive and negative infinity. The transmathematical result is that $0 / 0$ is nullity and $a / 0=1 / 0$ is positive infinity; positive because, in the Two Couriers problem, $a$ is a real numbered magnitude.

Just like in the case of standard mathematics, this is just enough information to distinguish Case 1 and Case 2, but not enough information to distinguish Case 2.1 and 2.2. The difference is that in transmathematics, $0 / 0$ and $1 / 0$ are numbers so a calculation in transmathematics distinguishes these cases, whereas, in standard mathematics the calculations both fail and we must engage in metamathematical reasoning to obtain an answer. In other words, the transmathematical solution is direct, whereas the standard mathematical result is indirect via metamathematics.

\subsection{Other Non-Standard Mathematics}

Both Saitoh [8] and Barukčić [4] use non-standard mathematical interpretations of division by zero. Saitoh defines $z / 0=0$, that is any division by zero is zero. Barukčić defines $0 / 0=1,1 / 0$ is positive infinity, and leaves it implicit that a negative number divided by zero is negative infinity. Thus Saitoh and Barukčić differ on the result of division by zero. Yet Barukčić defines non-zero divided by zero similar to transmathematics with an infinity as the result.

\subsubsection{Saitoh's $z / 0=0$}

For Saitoh, all cases of division by zero are zero.

One solution is that zero is at the start or origin. This might accidentally solve Case 2.1 but in Case 2.2 the two couriers are always together, not just at the origin, and in Case 1 the couriers are never together. Saitoh's arithmetic conflate all three of these cases so it fails to solve the Two Couriers Problem.

\subsubsection{Barukčić's $0 / 0=1, z / 0=$ infinity, for positive $z$}

Barukčić defines division by zero as unity, otherwise an infinity. Barukčić states explicitly that positive infinity is the result of a positive number 
divided by zero, leaving it implicit that a negative number divided by zero is negative infinity [4].

Similarly to Saitoh's arithmetic, with result zero, Barukčić's arithmetic, with zero divided by zero resulting in unity, might accidentally solve Case 2.1 but in Case 2.2 the two couriers are always together, not just at the point at unity, so Barukčić's arithmetic fails to solve the Two Couriers Problem. However, Barukčić's arithmetic is similar to transreal arithmetic in Case 1 that non-zero divided by zero is infinity indicates that the two couriers will never meet.

\section{Conclusion}

The Two Couriers Problem involves division by zero in an easily understood setting of the actual positions of two couriers. In this problem we have no doubt about what division by zero means in practical terms. The problem has two temporal solutions: Case 1, the couriers start off apart and remain apart forever; Case 2, the couriers remain together for all time. However, Case 2 devolves into two spatiotemporal solutions: in Case 2.1 the couriers remain together for all time at their single starting point; in Case 2.2 the two couriers remain together for all time by sweeping out a half-infinite line of points in space.

Saitoh's arithmetic has only one result for all cases of division by zero so it necessarily conflates all three cases of division by zero in the Two Couriers Problem. Saitoh's arithmetic has no explanatory power in the Two Couriers Problem.

All of the other arithmetics, considered here, have at least two different results for division by zero. They all have enough explanatory power to distinguish the temporal solutions Case 1 and Case 2 but they cannot distinguish the spatiotemporal Case 2.1 and Case 2.2. There are, however, differences between the arithmetics. Barukčić's arithmetic has $0 / 0=1$ which imposes the real solution that when the two couriers start off at the point at unity, they remain there for all time. This contradicts Case 2.2 so Barukčić's arithmetic cannot solve the Two Couriers Problem. Both standard mathematics and transmathematics distinguish the temporal Case 1 from Case 2 and are consistent with the actual position of the couriers but neither can distinguish the spatiotemporal Case 2.1 and Case 2.2. However, transmathematics has the advantage that temporal Case 1 and Case 2 can be distinguished by arithmetical calculation, whereas the calculations fail in standard mathematics, leaving it up to a more or less sophisticated argument in metamathematics to distinguish these cases. Thus transmathematics is the only arithmetic, considered here, that can calculate a temporal solution to the Two Couriers Problem.

\section{Acknowledgements}

Thanks are due to the anonymous translators who translated the brief excerpt from Clairaut and gave the insightful warning that the statement 
of the problem is ambiguous as to whether it relates to spatial, temporal or spatiotemporal solutions.

Thanks are also due to James Anderson for many helpful discussions during the preparation of this paper.

\section{References}

[1] On the Study and Difficulties of Mathematics . Taylor and Walton, London, 1837. See pp. 37-39.

[2] Basic Mathematics. Brooks/Cole, Belmont, California, 2010. See p. 61.

[3] James A. D. W. Anderson. Transmathematics. 2009.

[4] Jan Barukčić and Ilija Barukčić. Anti-aristotle - the division of zero by zero. Journal of Applied Mathematics and Physics, (4):749-761, 2016.

[5] Bittinger and Keedy. Basic Mathematics. Addison-Wesley, 7th edition, 1995. See p. 533.

[6] Alexis Claude Clairaut. Elémens d'algèbre. The Brothers Guerin, Paris, 1746. See pp. 20-30.

[7] Joseph Victor Collins. Advanced Algebra. American Book Company, 1913. See p. 226.

[8] Saburo Saitoh, Hiroshi Michiwaki, and Masato Yamada. Reality of the division by zero z $/ 0=0$. International Journal of Applied Physics and Mathematics, 6(1):1-8, 2016. 